\title{
A combined application of the boundary element method and Lobatto time-stepping procedure in 3-D partially saturated poroelastic problems
}

\author{
Andrey I. Petrov ${ }^{1, *}$, and Aleksandr A. Belov ${ }^{2}$ \\ ${ }^{1}$ Research and Education Center «Materials», Don State Technical University, 344000 \\ Rostov-on-Don, Russia \\ ${ }^{2}$ Research Institute for Mechanics, National Research Lobachevsky State University of Nizhni \\ Novgorod, 603950 Nizhny Novgorod, Russia
}

\begin{abstract}
The report presents a modification of a time-step scheme on the nodes of Runge-Kutta methods for solving 3-D dynamic poroelastic problems using the boundary element method. To describe poroelastic medium, Biot's mathematical model of a material is employed, in which solid phase and two fluid phases, filling the pores -liquid and gas, are discerned. 2- and 3-stage Lobatto methods were chosen as a particular example of Runge-Kutta methods. A comparison of the results obtained using the Lobatto method and the Euler method is presented.
\end{abstract}

\section{Introduction}

In BEM-modeling of dynamic processes, three main approaches can be conventionally discerned: solving in time domain [1], solving in Laplace or Fourier transforms with the subsequent inversion of the transforms [2], and the dual reciprocity approach [3]. The numerical accuracy of the time-domain BEM with time-stepping discretization is strongly influenced by the time step size. In particular, the time-marching process becomes numerically unstable when using collocation methods where the time step size is smaller than the element size. To overcome this difficulty, a number of stabilization techniques were developed $[4,5]$. However, the time-domain approach cannot be used to solve wave propagation problems for poroelastic media due to the absence of fundamental solutions in time. Methods working in the frequency domain also have their limitations, as they require efficient numerical techniques for inverse transforms and are applicable only to problems for which the correspondence principle is valid.

In 1988, Lubich [6,7] introduced the convolution quadrature method (CQM) to discretize the convolution integral. It gained significant interest as a technique (CQ-BEM) for applying the BEM to time-dependent problems where classical time-step schemes suffer from instability and numerical damping [8-10]. Later, it was shown in several studies that the CQ-BEM based on implicit Runge-Kutta provides better accuracy than that based on

*Corresponding author: andrey.petrov@mech.unn.ru 
the linear multistep method [11-15]. Also, Banjai and Sauter reformulated CQ-BEM approach to solve decoupled problems in Laplace domain that works as a transformation method [16]. Retaining the numerical stability properties of the original method, this approach has the time step size as the only parameter determined by the physical parameters of the problem. The applicability of the reformulated CQ-BEM to poroelastic problems was demonstrated by Schanz, but only using the linear multistep method [17].

The present study presents a modification of such a time-step scheme on the nodes of Runge-Kutta methods, exemplified by 2- and 3-stage Lobatto method. The time-step BEM scheme is based on the stepped method for numerically inverting Laplace transforms. The application of the scheme to problems of wave propagation in partially saturated media is considered.

\section{Mathematical model}

To describe a poroelastic continuum, Biot's mathematical model of a material is used, in which (the material) a solid phase, representing a form-generating skeleton, carrying the main stress load, and two fluid phases - water and air filling the pore system, are discerned. All the three phases are assumed to be compressible. Temperature variations are neglected. Besides, porosity $\varphi$ is defined as $\varphi=V_{\text {void }} / V$ and saturation degree of the material $S_{f}$ is defined as $S_{f}=V_{f} / V_{\text {void }}$, where $V_{\text {void }}$ is volume of interconnected pores in the specimen, $V$ is total volume of the material, $V_{f}$ is volume of the filler, and it is assumed that $f=a$ for air and $f=w$ for water. Consider a case where the pores are completely filled. The bulk density is defined as $\rho=(1-\varphi) \rho_{s}+\varphi S_{w} \rho_{w}+\varphi S_{a} \rho_{a}$, where the densities of the solid, water and air is denoted by $\rho_{s}, \rho_{w} \rho_{a}$, respectively.

In order to describe mechanical behavior of the partially saturated porous medium, the effective stress principle is used, which was introduced by Terzaghi [18]. The corresponding defining relations in terms of stress are expressed similarly to that adopted for fully saturated conditions:

$$
\sigma_{i j}=\left(K-\frac{2}{3} G\right) \varepsilon_{k k} \delta_{i j}+2 G \varepsilon_{i j}-\alpha\left(S_{a} p^{a}-S_{w} p^{w}\right) \delta_{i j},
$$

where $\sigma_{i j}$ is the total stress, $p^{a}$ denotes the pore air pressure, $p_{w}$ is the pore water pressure, $\alpha$ is the Biot constant, $G$ denotes the shear modulus, $\varepsilon_{i j}$ denotes the strain tensor. The coefficient $\alpha$ is described by the relationship $\alpha=1-K / K_{s}$, where $K$ is the bulk modulus of the solid skeleton and $K_{s}$ is the bulk modulus of the solid grains. The components of strain tensor $\varepsilon_{i j}$ of a solid and displacements $u_{i}$ are correlated by geometric relations $\varepsilon_{i j}=\left(u_{i, j}+u_{j, i}\right) / 2$.

To construct equations of motion, defining equations must be combined with the related balance equations of momentum and balance equations of mass of each of the phases (for details see [19]). Using a Laplace transform makes it possible to write down the dynamic equations of a poroelastic medium in the form of a boundary-value problem for unknown displacement functions of the elastic skeleton $\hat{u}_{i}$ and the pore pressures of the fillers $\hat{p}^{w}$ and $\hat{p}^{a}$ : 


$$
\begin{aligned}
& G \hat{u}_{i, j j}+(K+G / 3) \hat{u}_{j, i j}-\left(\rho-\beta S_{w} \rho_{w}-\gamma S_{a} \rho_{a}\right) s^{2} \hat{u}_{i}-(\alpha-\beta) S_{w} \hat{p}_{, i}^{w}-(\alpha-\gamma) S_{a} \hat{p}_{, i}^{a}=-\hat{F}_{i},(2) \\
& -(\alpha-\beta) S_{w} s \hat{u}_{i, i}-\left(\zeta S_{w w} S_{w}+\frac{\varphi}{K_{w}} S_{w}-S_{u} \varphi\right) s \hat{p}^{w}+\frac{\beta S_{w}}{\rho_{w} s} \hat{p}_{, i i}^{w}-\left(\zeta S_{a a} S_{w}+S_{u} \varphi\right) s \hat{p}^{a}=-\hat{I}^{w}, \\
& -(\alpha-\gamma) S_{a} s \hat{u}_{i, i}-\left(\zeta S_{w w} S_{a}+S_{u} \varphi\right) s \hat{p}^{w}-\left(\zeta S_{a a} S_{a}+\frac{\varphi}{K_{a}} S_{a}-S_{u} \varphi\right) s \hat{p}^{a}+\frac{\gamma S_{a}}{\rho_{a} s} \hat{p}_{, i i}^{a}=-\hat{I}^{a},
\end{aligned}
$$

where $K_{w}$ and $K_{a}$ are bulk moduli of the fluid, $\hat{F}_{i}, \hat{I}^{w}, \hat{I}^{a}$ are bulk body forces. Symbol “^» denotes Laplace transform with complex variable $s$.

The following abbreviations

$$
\begin{aligned}
& \zeta=\frac{\alpha-\varphi}{K_{s}}, S_{w w}=S_{w}-\vartheta\left(S_{w}-S_{r w}\right), S_{a a}=S_{a}+\vartheta\left(S_{w}-S_{r w}\right), \gamma=\kappa_{a} \varphi \rho_{a} s /\left(\varphi S_{a}+\kappa_{a} \rho_{a} s\right), \\
& S_{u}=-S_{e}^{\frac{\vartheta+1}{\vartheta}} \vartheta\left(S_{r a}-S_{r w}\right) / p^{d}, S_{e}=\left(S_{w}-S_{r w}\right) /\left(S_{r a}-S_{r w}\right), \beta=\kappa_{w} \varphi \rho_{w} s /\left(\varphi S_{w}+\kappa_{w} \rho_{w} s\right)
\end{aligned}
$$

are introduced, where $S_{r w}$ is the residual wetting fluid saturation and $S_{r a}$ is the non-wetting fluid entry saturation, $S_{e}$ denotes the effective wetting fluid saturation degree. The symbol $p^{d}$ is non-wetting fluid entry pressure, $\vartheta$ is the pore size distribution index. The phase permeability of the wetting and the non-wetting fluid are given by $\kappa_{w}=K_{r w} k / \eta_{w}$ and $\kappa_{a}=K_{r a} k / \eta_{a}$ respectively. $K_{r w}$ and $K_{r w}$ denotes the relative fluid phase permeability, $k$ denotes the intrinsic fluid permeability, $\eta_{w}$ and $\eta_{a}$ are viscosity of the fluid. To evaluate relative phase permeability following equations are used

$$
K_{r w}=S_{e}^{(2+3 \vartheta) / \vartheta}, K_{r a}=\left(1-S_{e}\right)^{2}\left(1-S_{e}^{(2+\vartheta) / \vartheta}\right) .
$$

\section{Laplace transform inversion method}

The stepped method for numerically inverting Laplace transforms is described in this section in brief. In general, the integral

$$
y(t)=\int_{0}^{t} f(\tau) d \tau
$$

is approximated as follows:

$$
y(0)=0, y(n \Delta t)=\mathbf{b}^{T} \mathbf{A}^{-1} \sum_{k=1}^{n} \boldsymbol{\omega}_{k}^{\Delta t}, n=1, \ldots N
$$

where $N$ is number of equal time steps. In this expression the quadrature weights $\boldsymbol{\omega}_{k}^{\Delta t}$ are determined using Laplace representation $\hat{f}(s)$ and the Runge-Kutta method. The quadrature weights can be expressed by the Cauchy integral form and approximated by using a trapezoidal rule with the number of steps $L$ as follows: 


$$
\boldsymbol{\omega}_{n}^{\Delta t}=\frac{R^{-n}}{L} \sum_{l=0}^{L-1} \hat{f}\left(\frac{\psi(z)}{\Delta t}\right) \frac{\psi(z)}{\Delta t} e^{-n l \frac{2 \pi}{L} \mathrm{i}}, z=R e^{l \frac{2 \pi}{L} \mathrm{i}}, \psi(z)=\mathbf{A}^{-1}-z \mathbf{A}^{-1} \mathbf{1 b}^{T} \mathbf{A}^{-1}
$$

where $\psi(z)$ is characteristic function of the Runge-Kutta method and $\mathbf{1}=(1,1, \ldots, 1)^{T}$. The parameter $R$ can be calculated by $R^{L}=\sqrt{\varepsilon}$, where $\varepsilon$ is the error of the numerical calculation of Eq. (10).

The approximation used in deriving formulas (17) is based on using the $m$-stage RungeKutta method written down using Butcher's table:

$$
\frac{\mathbf{c} \mid \mathbf{A}}{\mathbf{b}^{T}}, \mathbf{A} \in \mathbb{R}^{m \times m}, \mathbf{b}, \mathbf{c} \in \mathbb{R}^{m} .
$$

A correct formulation of a time-step scheme requires that the method be A-stable and Lstable. In the assumption of $\mathbf{b}^{T} \mathbf{A}^{-1}=(0, \ldots, 0,1)$, the method is automatically L-stable. Also it is important to note that the quadrature weights $\boldsymbol{\omega}_{k}^{\Delta t}$ and the characteristic function $\psi(z)$ are $m$-order matrices.

\section{BEM formulation and numerical procedure}

The boundary-element technique is based on the use of a regularized BIE direct approach:

$$
\int_{\Gamma}\left(\mathbf{T}(\mathbf{x}, \mathbf{y}, s) \mathbf{u}(\mathbf{x}, s)-\mathbf{T}^{\mathbf{0}}(\mathbf{x}, \mathbf{y}) \mathbf{u}(\mathbf{y}, s)\right) d \Gamma=\int_{\Gamma} \mathbf{U}(\mathbf{x}, \mathbf{y}, s) \mathbf{t}(\mathbf{x}, s) d \Gamma, \mathbf{x}, \mathbf{y} \in \Gamma, \Gamma=\partial \Omega,
$$

where $\mathbf{U}(\mathbf{x}, \mathbf{y}, s)$ and $\mathbf{T}(\mathbf{x}, \mathbf{y}, s)$ are matrices of fundamental and singular solutions, respectively, $\mathbf{T}^{\mathbf{0}}(\mathbf{x}, \mathbf{y})$ contains isolated singularities, $\mathbf{x}$ is integration point, $\mathbf{y}$ is observation point, $\mathbf{u}$ is generalized displacement vector, $\mathbf{t}$ is generalized force vector.

To solve equation (12), the boundary surface $\Gamma$ is divided into generalized eight-node quadrangular elements. Generalized boundary functions of the first kind are approximated bilinearly, and generalized boundary functions of the second kind are assumed to be constant over the element. The discrete representation of BIE (12) is constructed at the interpolation nodes of unknown boundary functions (collocation points) and has a matrix form, where the matrices contains integrals of the components of matrices $\mathbf{U}(\mathbf{x}, \mathbf{y}, s)$ and $\mathbf{T}(\mathbf{x}, \mathbf{y}, s)$, multiplied by the form functions. The choice of the numerical integration scheme for computing the integral depends on its type. When a collocation point lies on integration element, the procedure for revealing the feature is performed. To improve the accuracy of integration on an element that does not contain a collocation point, in addition to the Gauss integration formulas, a hierarchical integration algorithm is applied, wherein the element is subdivided until the specified accuracy is achieved.

\section{Results and discussion}

The $3 \mathrm{~d}$ poroelastic column loaded by a Heaviside function is investigated as example to study the behavior of transformation method [20]. The width of the column is $b=1 \mathrm{~m}$, the height $h=3 m$. The column has zero displacements on one end and on the other end the normal traction $t_{3}=-1 N / \mathrm{m}^{2}$ is prescribed. Three meshes of 224, 896 and 3584 
quadrangular boundary elements were used for calculation, respectively. The parameters of the partially saturated porous material correspond to those of sandstone.

The solution of the above problem is compared to the solution obtained earlier [20] on the basis of the time-step boundary-element scheme at the nodes of the Euler method. All boundary-element solutions are also compared with a one-dimensional numerical-analytical solution. The traction $t_{3}$ at the fixed end is chosen as compared values. Since the traction change spasmodically, this choice allows us to compare the results under additional approximation difficulties of the solution.

Fig. 1-2 presents the graphs of the tractions on the fixed end of the console. Fig. 1a shows that application of the 2- and 3-stage Lobatto methods makes it possible to obtain boundary-element solutions close to the numerical-analytical one already on the mesh with the smallest number of elements. A graphical comparison of the forces computed on different meshes using 2-stage method is shown in Fig. 2a: an increase in number of boundary elements makes it possible to improve the solution, but at the same time requires more complex-valued points for calculations, as well as changes in parameter of the step scheme $\Delta t$. When using 3-stage method, the refinement of the solution is also observed with an increase in number of elements from 224 up to 896 (Fig. 2b). However, further mesh refinement does not lead to significant improvements - the solutions are not graphically distinguishable. In addition, in this case all calculations are performed with the same value $\Delta t$. Nevertheless, despite a larger time step size, the solution at the nodes of the 3 -stage method is smooth, and the amplitude of the oscillations at the points of the wave front does not exceed the amplitude of oscillations of solutions at the nodes of the 2-stage method.
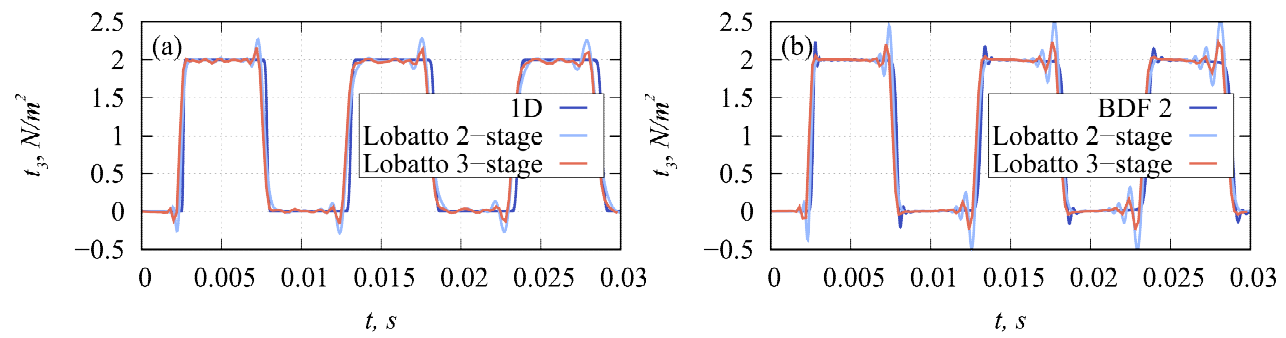

Fig. 1. Traction at the fixed end versus time: BEM and numerical-analytical solutions (a); solutions with different multistep methods (b).
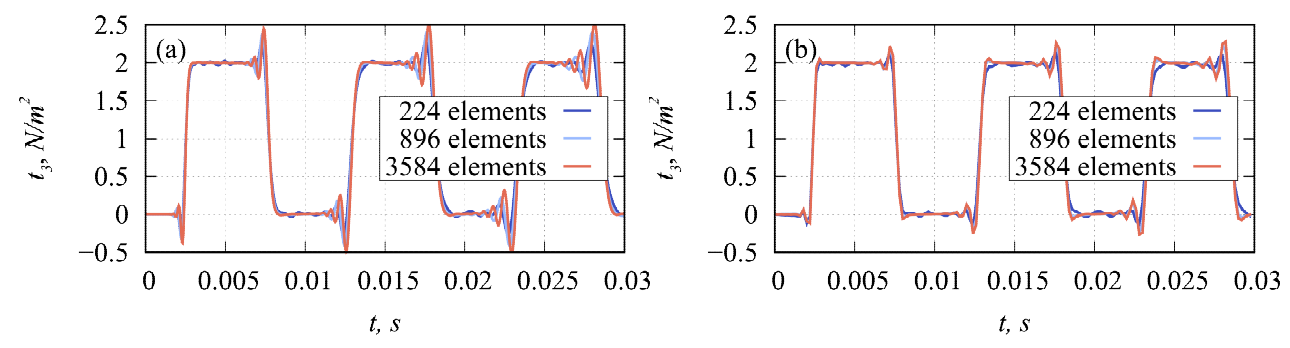

Fig. 2. Traction at the fixed end versus time: solutions with Lobatto 2-stage (a) and 3-stage (b) methods.

Comparison in Fig. $1 \mathrm{~b}$ shows good agreement between the results, obtained using the Lobbatto and the Euler schemes [20]. It's seen that the result of application of 3-stage Lobatto method in this task is comparable with the result of 2-stage Lobatto and Euler 
methods, but required less number of complex-valued points and the mash with less number of boundary elements and respectively less computational cost.

\section{Conclusion}

A time-step boundary-element scheme on the nodes of a family of Runge-Kutta methods for analyzing initial boundary-value problems of the dynamics of partially saturated poroelastic bodies has been considered. The problem of the effect of the force on the end of poroelastic prismatic body has been solved. The results of a numerical experiment obtained using 2- and 3-stage Lobatto schemes are presented. The comparison with the solution, obtained earlier using the Euler schemes is also presented. The boundary-element solutions are in good agreement with the numerical-analytical solution. The boundary-element solutions are in good agreement with the numerical-analytical solution. The results of numerical experiments showed that the use of 3-stage Lobatto method allows to reduce computing costs.

The research was carried out under the financial support of the Russian Scientific Foundation (project no. 15-19-10056).

\section{References}

1. W.J. Mansur, A Time-Stepping Technique to Solve Wave Propagation Problems Using the Boundary Element Method (University of Southampton, UK, 1983)

2. T.A. Cruse, F.J. Rizzo, J. Math. Anal. Appl., 22, 244 (1968)

3. M.H. Aliabadi, The boundary element method: applications in solids and structures (Wiley, London, 2002)

4. Y. Guoyou, W.J. Mansur, J.A.M. Carrer, L. Gong, Comput. Struct., 74, 495 (2000)

5. T. Ha-Duong, B. Ludwig, I. Terrasse, J. Numer. Methods Engrg., 57, 1845 (2003)

6. C. Lubich, Numer. Math., 52, 129 (1988)

7. C. Lubich, Numer. Math., 52, 413 (1988)

8. M. Schanz, H. Antes, Meccanica, 32, 179 (1997)

9. A.I. Abreu, J.A.M. Carrer, W.J. Mansur, Eng. Anal. Bound. Elem., 27, 101 (2003)

10. Ch. Zhang, Int. J. Solids Struct., 37, 6107 (2000)

11. C. Lubich, A. Ostermann, Math. Comp., 60, 105 (1993)

12. L. Banjai, M. Messner, M. Schanz, Comput. Methods Appl. Mech. Engrg., 245-246, 90 (2012)

13. M.P. Calvo, E. Cuesta, C. Palencia, Numer. Math., 107, 589 (2007)

14. L. Banjai, C. Lubich, BIT, 51, 483 (2011)

15. L. Banjai, C. Lubich, J.M. Melenk, Numer. Math. 119, 1(2011)

16. L. Banjai, S. Sauter, SIAM J. Numer. Anal. 47(1), 227 (2008)

17. M. Schanz, CMES Comput. Model. Eng. Sci. 58(2), 109 (2010)

18. K. Terzaghi, Theoretical Soil Mechanics (John Wiley and Sons, New York, 1943)

19. P. Li, M. Schanz, Time domain boundary element formulation for partially saturated poroelasticity. Eng. Anal. Bound. Elem. 37(11), 1483 (2013)

20. L.A. Igumnov, A.N. Petrov, PNRPU Mech. Bull. 3, 47 (2016) 